SLAC-PUB-11211

June 2005

\title{
DAMPING HIGHER ORDER MODES IN THE PEP-II B-FACTORY VERTEX BELLOWS*
}

\author{
S. Weathersby, J. Langton, A. Novokhatski, J. Seeman, Stanford Linear Accelerator
}

Center, Stanford University, Stanford, CA 94309

\begin{abstract}
Higher stored currents and shorter bunch lengths are requirements for increasing luminosity in colliding storage rings. As a result, more HOM power is generated in the IP region. This HOM power propagates to sensitive components causing undesirable heating, thus becoming a limiting issue for the PEP-II B-factory. HOM field penetration through RF shielding fingers has been shown to cause heating in bellows structures. To overcome these limitations, a proposal to incorporate ceramic absorbers within the bellows cavity to damp these modes is presented. Results show that the majority of modes of interest are damped, the effectiveness depending on geometrical considerations. An optimal configuration is presented for the PEP-II B-factory IR bellows component utilizing commercial grade ceramics with consideration for heat transfer requirements.
\end{abstract}

Contributed to Particle Accelerator Conference

Knoxville, Tennessee, USA

May 16 - May 20, 2005

*Work supported by Department of Energy contract DE-AC02-76SF00515. 


\section{DAMPING HIGHER ORDER MODES IN THE PEP-II B-FACTORY VERTEX BELLOWS*}

\begin{abstract}
S. Weathersby, J. Langton, A. Novokhatski, J. Se Abstract

Higher stored currents and shorter bunch lengths are requirements for increasing luminosity in colliding storage rings. As a result, more HOM power is generated in the IP region. This HOM power propagates to sensitive components causing undesirable heating, thus becoming a limiting issue for the PEP-II B-factory. HOM field penetration through RF shielding fingers has been shown to cause heating in bellows structures. To overcome these limitations, a proposal to incorporate ceramic absorbers within the bellows cavity to damp these modes is presented. Results show that the majority of modes of interest are damped, the effectiveness depending on geometrical considerations. An optimal configuration is presented for the PEP-II B-factory IR bellows component utilizing commercial grade ceramics with consideration for heat transfer requirements.
\end{abstract}

\section{INTRODUCTION}

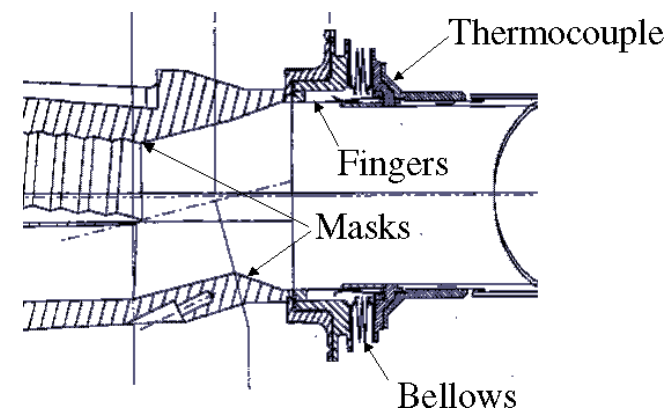

Figure 1: IP vertex chamber view.

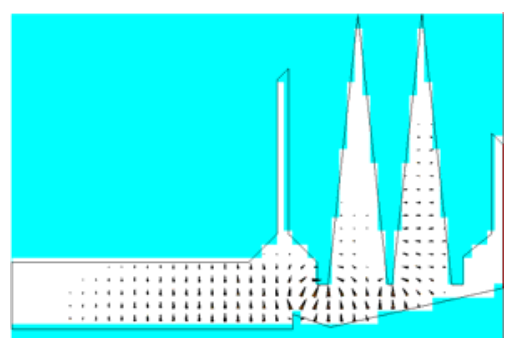

Figure 2: Bellows cavity cross section with a $5.3 \mathrm{GHz}$ mode.

The PEP-II B-factory collides $3 \mathrm{GeV}$ positrons with 10 $\mathrm{GeV}$ electrons in the BaBar detector IP region. Run 4 achieved production luminosities of $9 \times 10^{33} / \mathrm{cm}^{2} \mathrm{~s}$ at currents of $2.4 \mathrm{~A}$ positrons in the low energy ring (LER) and

\footnotetext{
* Work supported by the U.S. Department of Energy under contract number DE-AC03-76SF00515.
}

1.5 A electrons in the high energy ring (HER) in the form of bunch trains comprising typically 1600-1700 $1.3 \mathrm{~cm}$ long bunches. At these currents and bunch lengths various beam line components experience anomolous heating attributed to higher order beam induced electromagnetic fields (HOMs). Temperature measurements indicated there was sufficient heating to damage critical components in the IP region which sees the effect of both beams simultaneously. Based on thermocouple data estimates[1] of $0.5 \mathrm{~kW}$ of HOM power is coupling into the IP bellows at these currents. Upwards of $2 \mathrm{~kW}$ is expected at the projected 3-4 A currents.

Figure 1 is a drawing of the IP region where the incoming positron beam chamber (outgoing electron) from the left side connects to the beryllium chamber of the BaBar detector to the right. This view reveals a nonuniform cross section of synchrotron masks, tapers and offsets which beams must traverse.

During a recent down time additional air and water cooling was installed enabling higher current collisions[2]. In order to substantially reduce the heating problem it is necessary remove HOM power from the bellows cavity. This power will increase quadratically with currents and inversely with the square of the bunch length. The best way to remove HOM power is to incorporate a water cooled absorber in the bellows cavity. The HOM power will be captured by the absorber and removed by water cooling. We suggest a new IP bellows design based on this approach.

\section{PHYSICAL PROCESSES CONTRIBUTING TO HOM HEATING}

Irregularities in the vacuum chamber which can disturb the beam fields contribute to the generation of higher order modes. Reflected and scattered waves are generated by impedance producing elements in the ring such as collimators, masks, tapers and crotch areas where two beamlines intersect. These waves manifest transverse components which can propagate some distance and couple into sensitive beam line components such as bellows assemblies and anti-chambers. Particular volumes such as the interior of a bellows behave like cavities with substantial enough quality factor to trap a damaging amount of HOM power. This is particularly true for the IP region where many impedance producing elements are concentrated.

Bunch length and bunch pattern strongly dictate the degree to which beam line elements contribute to the impedance spectrum. The push to lower bunch lengths will shift the power distribution to higher frequencies. In addition, bunch patterns can generate frequencies which couple strongly with beam line component resonances. For 
the $1.3 \mathrm{~cm}$ bunch length most beam power is generated at around the $4 \mathrm{GHz}$, however, there is sufficient power in the high frequency tail of the beam spectra to produce HOMs. Peaks at higher frequencies are observed to correlate with bellows heating. This high frequency power can couple into the bellows through the bellows fingers [3]. For the PEP-II IR vertex bellows HOM power couples to a bellows mode of about $5 \mathrm{GHz}$.

\section{EXISTING BELLOWS MODES AND $Q$ FACTORS}

\section{$Q$ Factor Calculations}

For material parameters we assume a stainless steel vacuum chamber conductivity. For the absorber we use parameters of a commercially available ceramic with relative permittivity $\epsilon=30$ and a loss factor $\tan \delta_{l}=0.11$. We present the calculated $Q$ factors for the existing structure in figure 2 without any absorber and three test cases involving absorbers in different geometries. The MAFIA[6] mesh generator, eigenmode solver and post processing modules were used to generate the data.

\section{Bellows Modes}

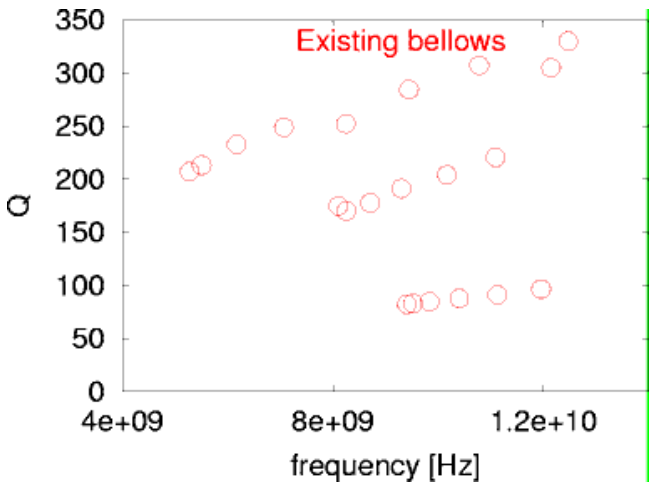

Figure 3: Existing bellows $Q$ factors as a function of frequency.

As a control for these studies $Q$ factors were determined for the existing undamped PEP-II IP vertex chamber. Figure 2 shows one of several bellows eigenmodes for the existing bellows strucure. For each of twenty of these modes a quality factor $(Q$ factor) is determined. The results are shown in figure 3 . The undamped $Q$ factors vary from 50 to several hundred over 5 to $12 \mathrm{GHz}$. The main concerns are low frequency high $Q$ modes. Coupling coefficient of $5 \times 10^{-3}$ provides perfect transmission of the power of transverse fields from the vacuum chamber to the bellows cavity through the shield fingers. Power is dissipated in the stainless steel surface.

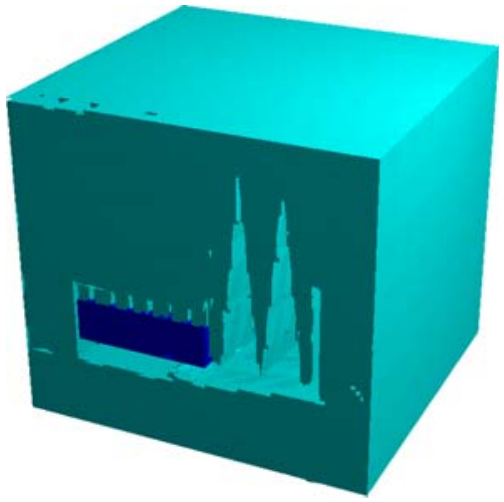

Figure 4: MAFIA mesh for large aspect absorber. Blue material is a ceramic absorber braised to vertical support columns.

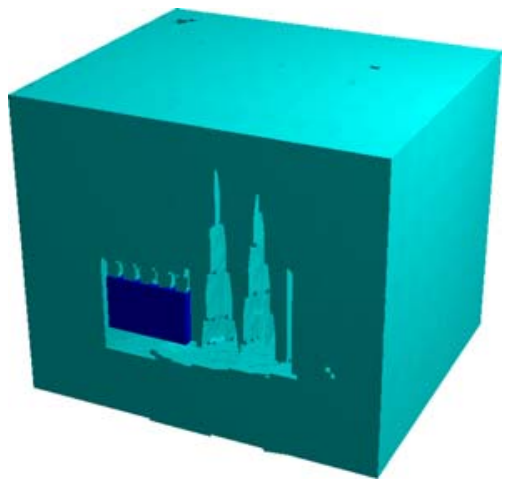

Figure 5: MAFIA mesh for small aspect sbsorber. Blue material is a ceramic absorber braised to vertical support columns.

\section{EFFECT OF A CERAMIC ABSORBER IN THE VERTEX BELLOWS}

Several prototypes were investigated with different absorber locations and geometries within the bellows cavity. Three candidate designs for a new vertex bellows chamber are shown in figures 7, 5 and 4 . They incorporate additional volume to house and support the absorber. Sup-

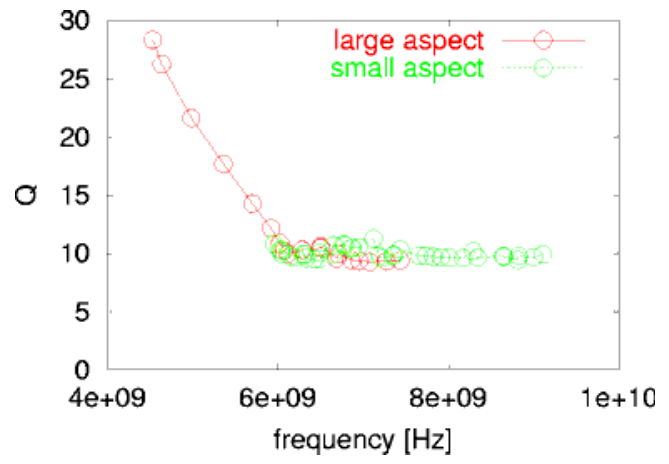

Figure 6: Comparison of damping for the large and small apect absorber configurations 
port columns allow for thermal expansion and heat transfer. The absorber is located above the fingers where lower frequency modes are the highest intensity and there is sufficient thermal contact with the outer chamber wall for cooling to be effective.

A comparison of damping effectiveness between the large and small aspect ratio absorber configurations of figures 5 and 4 is shown in figure 6 . Both configurations are effective in damping all modes. Although the small aspect configuration is preferable in this study, as a practical matter, the consideration of heat dissipation became a determining factor. A thermal heat flow analysis indicated insufficient heat dissipation from the small aspect absorber configuration due to its thickness and smaller thermal contact area with the braised support columns. This suggested the large aspect configuration would be a practical compromise.

Figures 5and 7 are essentially the same geometry with the exception of how the absorbers are supported. Figure 7 has horizontal support columns whereas the figure 5 support columns are vertical. The support column orientation differences have little effect on the damping as evident in figure 8.

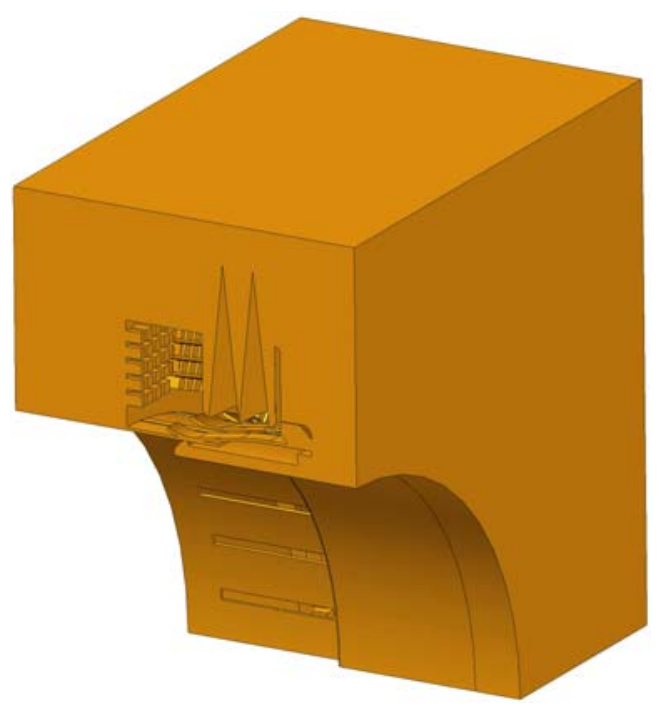

Figure 7: CAD view without the absorber showing horizontal support columns for the small aspect absorber test case.

The effective damping of this method can be seen in $Q$ factor calculations on these structure with and without the influence of the ceramic. This comparison is shown in figure 9. There are two main effects which are obvious from the plot. First and foremost is the order of magnitude reduction of the $Q$ factors due to the large loss factor. Secondly, mode frequencies are shifted down and condensed into a smaller range due to the large permittivity.

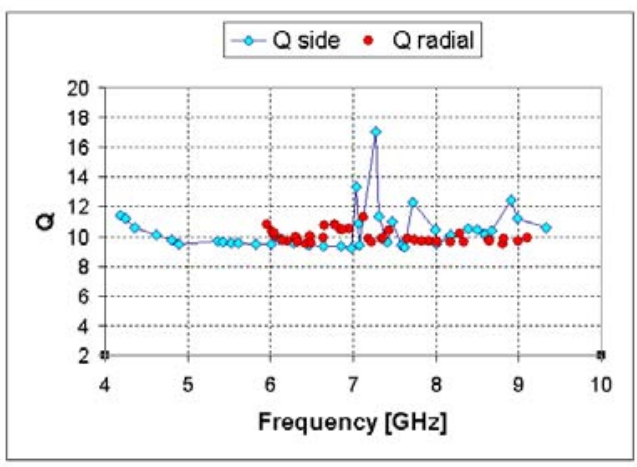

Figure 8: Effect of support column orientation. $Q$ factors for the geometry of figures 5 and 7 .

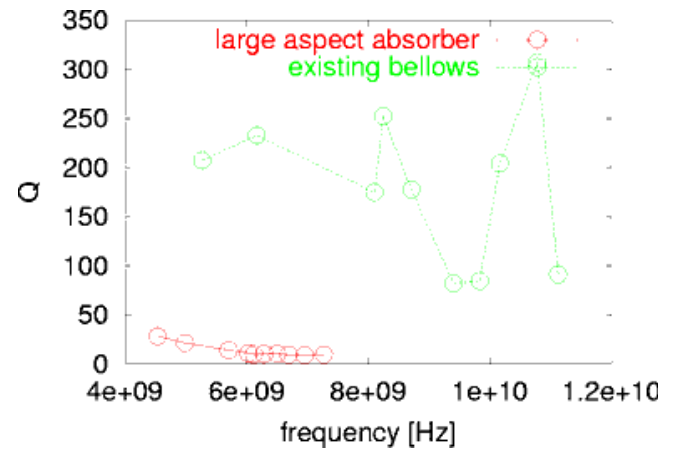

Figure 9: Effect of absorber on vertex bellows $Q$ factors. $Q$ factors for the undamped existing bellows and the large aspect absorber configuration (figure 4)

\section{CONCLUSIONS}

PEP-II has experienced HOM heating of bellows during high current operations. This has been a limiting performance issue for the B-factory. This study presents a means to reduce HOM heating in the IP region bellows for the anticipated higher current operation. Significant damping of HOMs with ceramic absorbers is achieved in a bellows while allowing adequate heat flow to remove HOM power in a controlled way.

\section{REFERENCES}

[1] Stan Ecklund, Private communication.

[2] J. Seeman, "Performance of the PEP-II e+e- Collider at SLAC', PAC'05, May 2005, these proceedings.

[3] A. Novokhatski, S. Weathersby, "RF MODES IN THE PEPII SHIELDED VERTEX BELLOWS”, PAC'03, May 2003.

[4] A. Novokhatski, S. Weathersby, "RF Heating in the PEP-II B-Factory Vertex Bellows”, ICAP'02, October 2002.

[5] F.Decker, S.Ecklund, A.Fisher, A. Kulikov, M. Sullivan, "Higher Order Mode Heating Observations in the PEP-II Interaction Region”, PAC'01, August 2001.

[6] The MAFIA collaboration, "User Guide" CST GmbH, Darmstadt, Germany. 